\title{
An approach for identifying and locating pavement sections subject to high dynamic wheel loads
}

\author{
R. A. Hassan \\ Swinburne University of Technology, Australia
}

\begin{abstract}
A study was conducted to assess the effectiveness of the International Roughness Index (IRI) and Heavy Articulated Truck Index (HATI) to identify pavement sections subject to high dynamic wheel loads (DWL). HATI is a profile-based roughness index used by some Australian road agencies to identify road sections providing poor ride quality for heavy trucks. The longitudinal surface profile data of ten pavement sections were used to simulate DWL caused by a typical fully laden articulated truck and to calculate relevant IRI and HATI values. The results of this limited study showed that HATI could be a good indicator of road sections subject to high DWL. For the sections tested herein, it was found to have strong correlation with the Dynamic Aggregate Force Coefficient (DAFC), a measure of road damage due to DWL. The study also included using wavelet analysis to identify and locate the sources of DWL excitation for sections with different HATI values. Wavelet signals of longitudinal profile data in relevant roughness subbands proved to be effective in identifying and locating profile characteristics responsible for exciting truck body motions that induce high DWL. The approach proposed herein would help road agencies to adopt maintenance programs to eliminate or minimise the additional damage and ensure sustainability of their assets' and mobility of road users.

Keywords: pavements, dynamic wheel loads, HATI, IRI, DAFC, wavelet analysis.
\end{abstract}

\section{Introduction}

It has been proven in many past studies [1-5] that the interaction between road surface roughness and heavy vehicle body results in dynamic wheel loads 
(DWL), which increase pavement damage. The magnitude of DWL is dependent on road surface profile characteristics which have a determining influence on dynamic loading and the degree of their spatial repeatability (Sweatman and Addis [6]). It is also dependent on travel speed and heavy vehicle properties, particularly the types of suspension systems. Considering the different available suspension and vehicle types, the excitation frequencies of DWL generally fall into two ranges, low and high. The low frequency range is $1.5-4 \mathrm{~Hz}$, which corresponds to rigid body bounce and pitch modes. The high frequency range is 8-15 Hz and corresponds to rigid body hop mode, axle roll and load sharing suspension pitch modes [2, 3]. At highway speeds along major freight routes $(80-100 \mathrm{~km} / \mathrm{hr})$ in Victoria / Australia, longitudinal road profile wavelengths that cause excitations of DWL at low and high frequencies fall in the range of (5.6$18.5 \mathrm{~m})$ and $(1.5-3.5 \mathrm{~m})$ respectively. However many studies [2-5] have shown that the lower frequency vibration modes are the major contributors to DWL for all suspension types.

The additional damage caused by DWL is reflected in the changes of surface profile characteristics so it is reasonable to assume that a suitable profile-based index could be used to reflect pavement functional condition and highlight sections that are subject to high DWL. This paper provides the details of a study on assessing the suitability of Heavy Articulated Truck Index (HATI), a profilebased index, as an indicator of the extent to which pavements are dynamically loaded. HATI is a pavement functional condition measure that highlights sections providing unpleasant ride to heavy vehicle occupants (Hassan and McManus [7]). The International Roughness Index (IRI) is also included in the assessment for comparison. The filters of these two indices are sensitive to the above roughness wavelengths but with different gains (i.e. amplification levels), hence it was hypothesised that they could be good measures for identifying dynamically loaded pavement sections, at network level. The study also includes an assessment of wavelet analysis, at project level, for locating and identifying sources of DWL excitations along a pavement section.

\section{Data preparation}

Ten sections were selected from three major freight routes in rural Victoria and range in length between 100 and $600 \mathrm{~m}$. All ten sections had flat and straight geometric alignments and were not in the vicinity of bridge abutments. Their cross sections were of a rural type with shoulders and table drains. They were built on different subgrade soil types, including expansive soils with different reactivity levels and subject to different climatic conditions. Pavements of these sections consist of thin granular base and subbase layers with sprayed seal wearing course. The longitudinal surface profile data of the ten sections were used as the excitation input for computer simulation of DWL and were also used to calculate sections' HATI and IRI values. The assessment involved performing correlation analyses of the above profile-based indices with a measure representing the extent of simulated DWL and associated damaging potential, namely, the Dynamic Aggregate Force Coefficient (DAFC) (Cebon [2]). Details 
of the simulation analysis, DAFC and HATI are provided in the following sections.

\subsection{Simulation of DWL}

The simulation was conducted using a full vehicle model of a five-axle tractorsemi-trailer combination unit. It had tandem drive and trailer axles with dual tyres and both were fitted with air bag suspensions. The vehicle was fully laden with a gross mass of 39 tonnes. It complies with Australian heavy vehicle mass and dimensions regulations and constitutes a reasonable proportion of the truck fleet in Victoria. The simulation speed was $100 \mathrm{~km} / \mathrm{hr}$, which is the speed limit for trucks on rural highways in Victoria. The simulation results included the distribution of dynamic wheel forces associated with all axles in the outer and inner wheel paths, sampled at 0.005 second intervals.

The DWL spectra of all the sections showed that the highest peaks occurred below $1 \mathrm{~Hz}(0.4-0.8 \mathrm{~Hz})$, which are believed to result from excitation of the body roll motion. The second set of high peaks occurred in the range of 1.5-2.5 $\mathrm{Hz}$ (frequencies corresponding to body bounce and pitch motions) and smaller amplitude peaks were also found in the $8-12 \mathrm{~Hz}$ range (corresponding to axle bounce motions). The low frequency modes are excited by the long wavelengths of the roughness spectrum and the high frequency ones are excited by short wavelengths. The excitation of the roll motion is related to the effects of the variation in road crossfall and the differences in elevation and direction of movement between the outer and inner wheel paths. The latter is true for all the sections tested herein and could be the source for the observed roll motion excitation.

\subsection{DWL road damage measure}

The dynamic wheel forces generated along each section by the tyres of all five axles, in both wheel paths, were used to estimate the DAFC. The DAFC is a measure of road damage caused by dynamic forces, normalised by the damage due to static forces. Since the spatial distribution of tyre forces is important, road damage criteria that relate to specific points along the road surface are needed for assessing road damage (Cebon [2]). The aggregate tyre force history of a vehicle is the sum of dynamic forces generated by all tyres applied to each location along the section, in both wheel paths, raised to a power (n) (eqn (1)) (Cebon [2]). Where $\mathrm{a}(\mathrm{t})$ is the aggregate force history of a vehicle with $\mathrm{N}$ axles; fk (t) is the tyre force history of axle $\mathrm{k}, \mathrm{k}=1,2,3 \ldots \mathrm{N}$; $\mathrm{N}$ is the number of axles. The recommended power is four $(n=4)$ when fatigue of asphalt is the dominant failure mode and one $(n=1)$ when permanent deformation (rutting) is the dominant failure mode. As the test sections are all made of flexible granular pavements, a power of one was used since rutting is considered to be their dominant failure mode.

$$
a(t)=\sum_{k=1}^{N} f_{k}(t)^{n}
$$


The DAFC is a measure that characterises the dynamic variation of the aggregate force. It is calculated as the ratio of the standard deviation of the aggregate tyre forces to their mean force (eqn (2)) (Cole and Cebon [8]).

$$
D A F C=\frac{\operatorname{std}[a(t)]}{\operatorname{mean}[a(t)]}
$$

\subsection{Heavy Articulated Truck Index (HATI)}

HATI is a profile-based index used by some Australian State Road Agencies for identifying pavement sections providing poor ride quality for heavy vehicle drivers. HATI is a distance-domain index that is suitable for network level management. It is determined by processing the longitudinal surface profile data of a section through a quarter truck filter. Details of HATI, its development and validation can be found in Hassan and McManus [7]. HATI captures the vertical motions associated with excitation of the low frequency body bounce and pitch vibration modes due to its sensitivity to long wavelength roughness. The highest gain $(>0.9)$ occurs in the frequency range 2-3.5 Hz, which can be excited by roughness wavelengths $8-14 \mathrm{~m}$ when travelling at $100 \mathrm{~km} / \mathrm{hr}$ (wavelength $(\mathrm{m})=$ speed $(\mathrm{m} / \mathrm{s}) /$ frequency $(\mathrm{Hz}$, cycle/sec)). HATI was developed using a mix of prime-mover semi-trailer combination units with different ages, suspension systems and other properties [7].

\section{Correlation analysis}

The DAFC values for each axle group and the whole vehicle together with the averages of HATI and IRI values of both wheel paths were determined for the ten sections and presented in table 1 . The DAFC values for the test sections are within the ranges reported by others for well damped air suspensions (Cebon [2]). Lower values of this measure mean better performance or lower damaging potential. For all the sections, DAFC values for the whole vehicle are lower than for the individual axle groups. This could be related to the averaging effect and load transfer by pitching from one axle to another (Bernard and Dolcemascolo [9]). It ranks the drive axle group as more damaging than the trailer axle group but similar to or more damaging than the steer axle.

The variation in HATI and IRI values for the test sections are the result of the considerable variation in their spectral properties. They vary in their roughness contents in the long and short wavebands of the roughness spectrum. Most of the test sections with high HATI values are built on highly reactive subgrade soils and located in climatic conditions that promote differential soil movement and the development of long wavelength roughness (LWR). Pavements built on soft subgrade soils have also shown to be associated with LWR due to differential settlement (Doré et al. [10]). 
Table 1: $\quad$ DAFC, HATI and IRI values for the test sections.

\begin{tabular}{|c|c|c|c|c|c|c|c|c|c|c|}
\hline Section No. & 1 & $\mathbf{2}$ & 3 & 4 & 5 & 6 & $\mathbf{7}$ & 8 & 9 & $\mathbf{1 0}$ \\
\hline Length, m & 500 & $\mathbf{6 0 0}$ & 500 & 500 & 200 & 500 & $\mathbf{3 0 0}$ & 300 & 100 & $\mathbf{2 0 0}$ \\
\hline DAFC Steer & 0.03 & $\mathbf{0 . 0 2}$ & 0.03 & 0.03 & 0.04 & 0.05 & $\mathbf{0 . 0 4}$ & 0.05 & 0.03 & $\mathbf{0 . 0 7}$ \\
\hline DAFC Drive & 0.03 & $\mathbf{0 . 0 2}$ & 0.03 & 0.03 & 0.04 & 0.06 & $\mathbf{0 . 0 4}$ & 0.06 & 0.03 & $\mathbf{0 . 0 6}$ \\
\hline DAFC Trailer & 0.02 & $\mathbf{0 . 0 1}$ & 0.02 & 0.02 & 0.02 & 0.04 & $\mathbf{0 . 0 3}$ & 0.04 & 0.02 & $\mathbf{0 . 0 5}$ \\
\hline DAFC (vehicle) & 0.01 & $\mathbf{0 . 0 1}$ & 0.02 & 0.01 & 0.02 & 0.02 & $\mathbf{0 . 0 2}$ & 0.03 & 0.02 & $\mathbf{0 . 0 3}$ \\
\hline IRI, m/km & 1.7 & $\mathbf{1 . 7 3}$ & 2.56 & 2.57 & 2.81 & 2.85 & $\mathbf{3 . 1 4}$ & 3.75 & 3.8 & $\mathbf{5 . 1 8}$ \\
\hline HATI, m/km & 1.06 & $\mathbf{0 . 5 8}$ & 1.37 & 1.11 & 1.24 & 1.83 & $\mathbf{1 . 4 4}$ & 2.08 & 1.54 & $\mathbf{3 . 0 9}$ \\
\hline
\end{tabular}

To increase the sample size, the profile data of each test section was divided into $100 \mathrm{~m}$ subsections resulting in a total of thirty-seven (37) subsections. The values of their profile indices in both wheel paths and their averages were then calculated. The original DWL signals (load vs. time) of the ten test sections were divided into 3.6 seconds segments (equivalent to $100 \mathrm{~m}$ ) and their DAFC values were also determined. Correlation analysis was performed to establish which of the two profile-based indices could be used to identify pavement sections subject to high dynamic wheel loads. The results of correlation analysis between HATI, IRI and DAFC are presented in table 2. The results clearly indicate that HATI is a better indicator of dynamically loaded sections since it has higher correlation coefficients (0.95 and 0.92$)$ than the IRI $(0.81$ and 0.78$)$. The good correlations of DAFC with these profile indices clearly indicate that there is contribution from both short and long roughness wavelengths. However, the higher correlation with HATI indicates higher contribution from the latter.

There is no indication in the available relevant literature of what DAFC value is acceptable. Hassan and McManus [7] proposed a threshold value for HATI of $2.2 \mathrm{~m} / \mathrm{km}$, which corresponds to a poor ride, to trigger investigation into pavement rehabilitation to improve ride quality for heavy truck occupants. This value can be used as an interim threshold for identifying dynamically loaded sections.

Table 2: Correlation coefficients between HATI and IRI with DAFC and wavelet energies in different subbands ( $\mathrm{n}=$ number of sections).

\begin{tabular}{|c|c|c|c|c|c|c|c|c|c|}
\hline & \multirow{2}{*}{$\begin{array}{c}\text { DAFC } \\
\end{array}$} & \multirow{2}{*}{$\begin{array}{c}\text { DAFC } \\
(\mathrm{n}=10)\end{array}$} & \multicolumn{5}{|c|}{ Wavelet energies in subbands D3-D9 $(\mathrm{n}=10)$} \\
\cline { 4 - 10 } & & Ed3 & Ed4 & Ed5 & Ed6 & Ed7 & Ed8 & Ed9 \\
\hline HATI & 0.95 & 0.92 & -0.49 & -0.14 & 0.36 & $\mathbf{0 . 8 8}$ & $\mathbf{0 . 8 1}$ & $\mathbf{0 . 7 7}$ & 0.02 \\
\hline IRI & 0.81 & 0.78 & -0.54 & -0.12 & 0.35 & 0.73 & 0.54 & 0.57 & -0.21 \\
\hline
\end{tabular}

\section{Wavelet analysis}

Wavelet analysis was used to identify and locate the sources of DWL excitation along the test sections. Discrete wavelet transform (DWT) decomposes the road profile signal into a number of wavebands and has been used in a number of studies to evaluate road roughness characteristics [11-14]. DWT was performed using a Matlab code to decompose the longitudinal profile data of the test sections into different subbands. A Daubechies wavelet of the order of seven 
(DB7) was used. The level of DWT decomposition depends on the length of the profile and its sample interval. For a $100 \mathrm{~m}$ section with $0.05 \mathrm{~m}$ sampling interval, there are 10 levels of decomposition and the ranges of wavelengths in each level or subband (D) are: D1 (0.1-0.2 m), D2 (0.2-0.4 m), D3 (0.4-0.8 m), D4 (0.8-1.6 m), D5 (1.6-3.2 m), D6 (3.2-6.4m), D7 (6.4-12.8m), D8 (12.825.6m), D9 (25.6-51.2m) and D10 (51.2-102 m). For the ten sections tested herein, the maximum level of decomposition ranged between 10 and 13 .

DWT analysis output included the signals (elevation vs. distance) and energies (a measure of elevation variation) in the different subbands. HATl values of the sections were correlated with the corresponding energies (Ed) in the subbands (D3-D9) covering wavelengths related to surface roughness (0.5-50 $\mathrm{m})$. It can be noticed in table 2 that the subbands with the highest coefficients are D6, D7 and D8. The signals of these subbands, in both wheel paths for three test sections were used to identify and locate the sources of DWL excitations along their lengths. The sections were selected to have low, medium and high HATI values and included sections 2, 7 and 10 in table 2 respectively.

The difference between the signals of the two wheel paths in any subband represents a roll excitation input and their average represents a bounce excitation input. The signals of roll and bounce excitation inputs in subbands D6, D7 and D8 for sections 2, 7 and 10 are presented in figures 1,2 and 3 respectively together with corresponding DWL signals. The latter is presented in terms of the ratio of total vehicle DWL (both wheel paths) to static load at each point along the length of the section. Careful examination of the three figures revealed the following:

- Generally, the concentration of profile signals energies and peaks in the wavebands 3-26 m (subbands D6 to D8) match with DWL signals variations, keeping in mind that there is a shift between the source of DWL excitation and its impact. The DWL signal combines the contributions of all axle groups, with variable shifts in impacts and contributions along the section. A summary of general observations is provided below.

o Section 2: The highest DWL occurs between 470 and $570 \mathrm{~m}$ and the signals in the three subbands (D6 to D8) show clear concentration of roll and bounce inputs, higher from the first. This is also true for the DWL concentration between 200 and $300 \mathrm{~m}$.

o Section 7: The highest DWL occurs at the start of the section, 0$35 \mathrm{~m}$ due to bounce and roll inputs (higher from the first) that are evident in the three subbands. The next peak is between $230-240 \mathrm{~m}$ and the excitation inputs are evident in all three subbands.

o Section 10: The highest DWL occurs between 10 and $30 \mathrm{~m}$ and the signals in the three subbands, more evident in D6 and D7, clearly indicate a concentration of input from bounce and roll. The latter also corresponds to the concentration of DWL between 90 and $140 \mathrm{~m}$. 


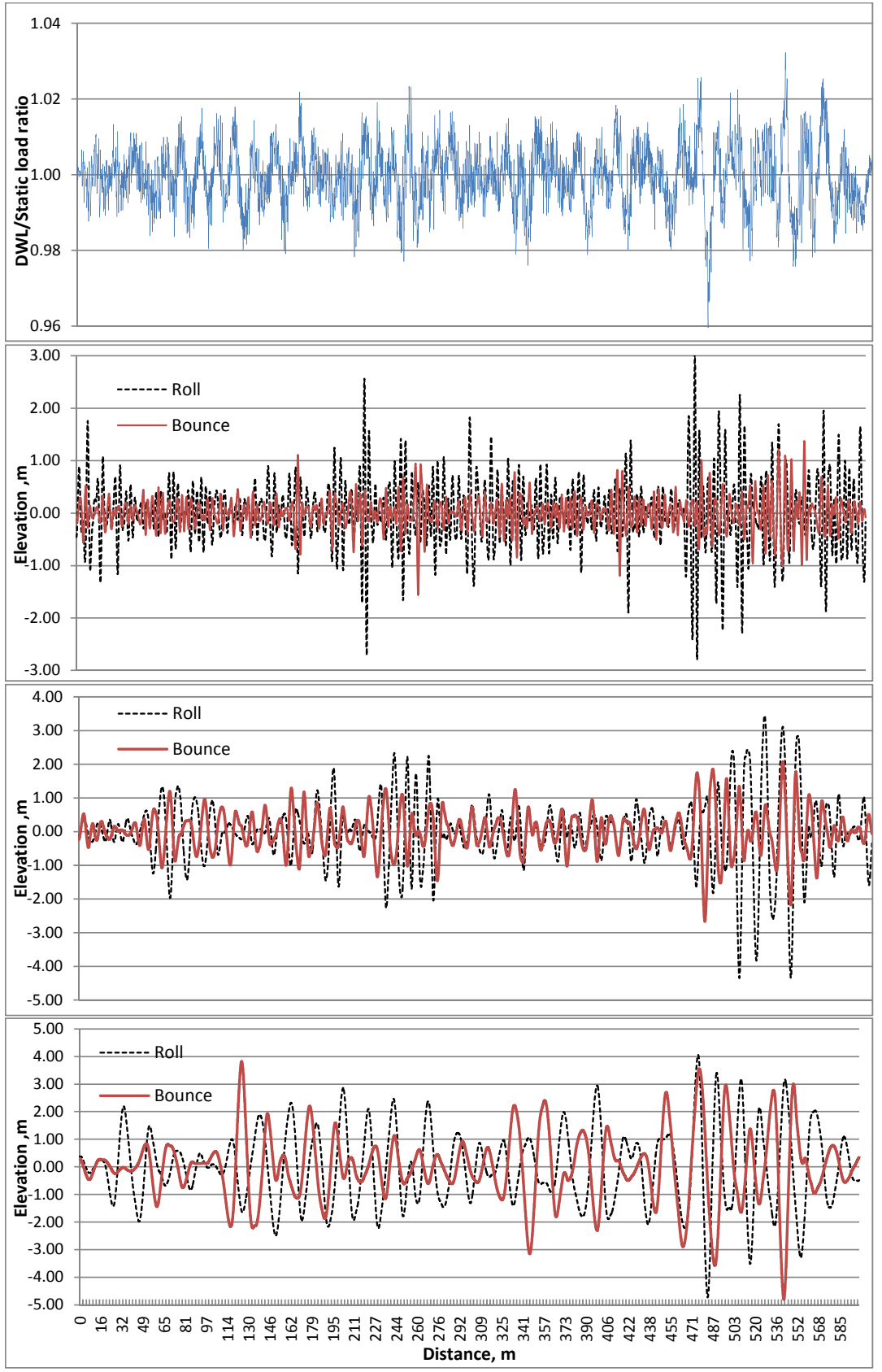

Figure 1: DWL to static load ratio and roll and bounce excitation inputs in D6, D7 and D8, respectively for section 2. 


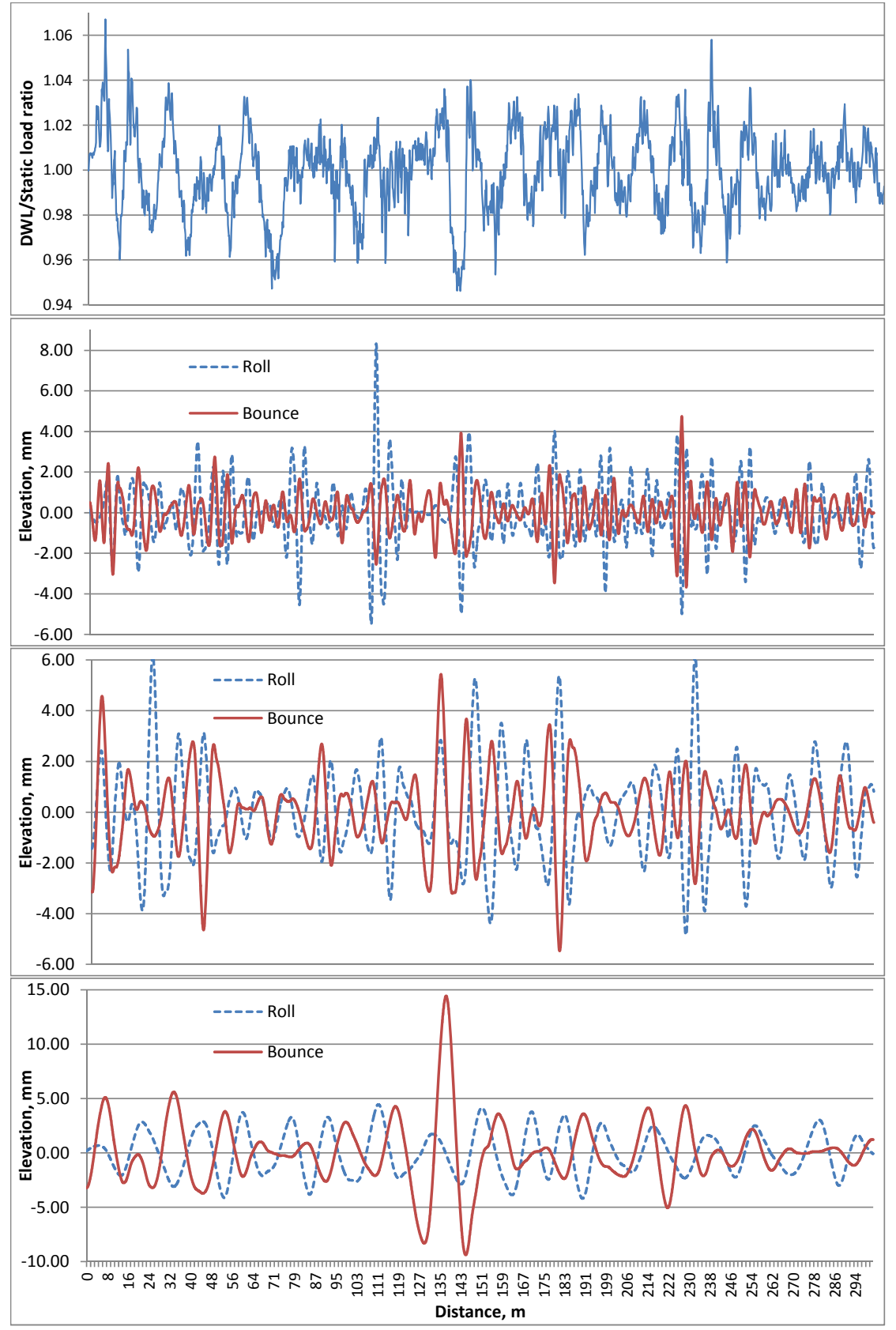

Figure 2: DWL to static load ratio and roll and bounce excitation inputs in D6, D7 and D8, respectively for section No. 7. 


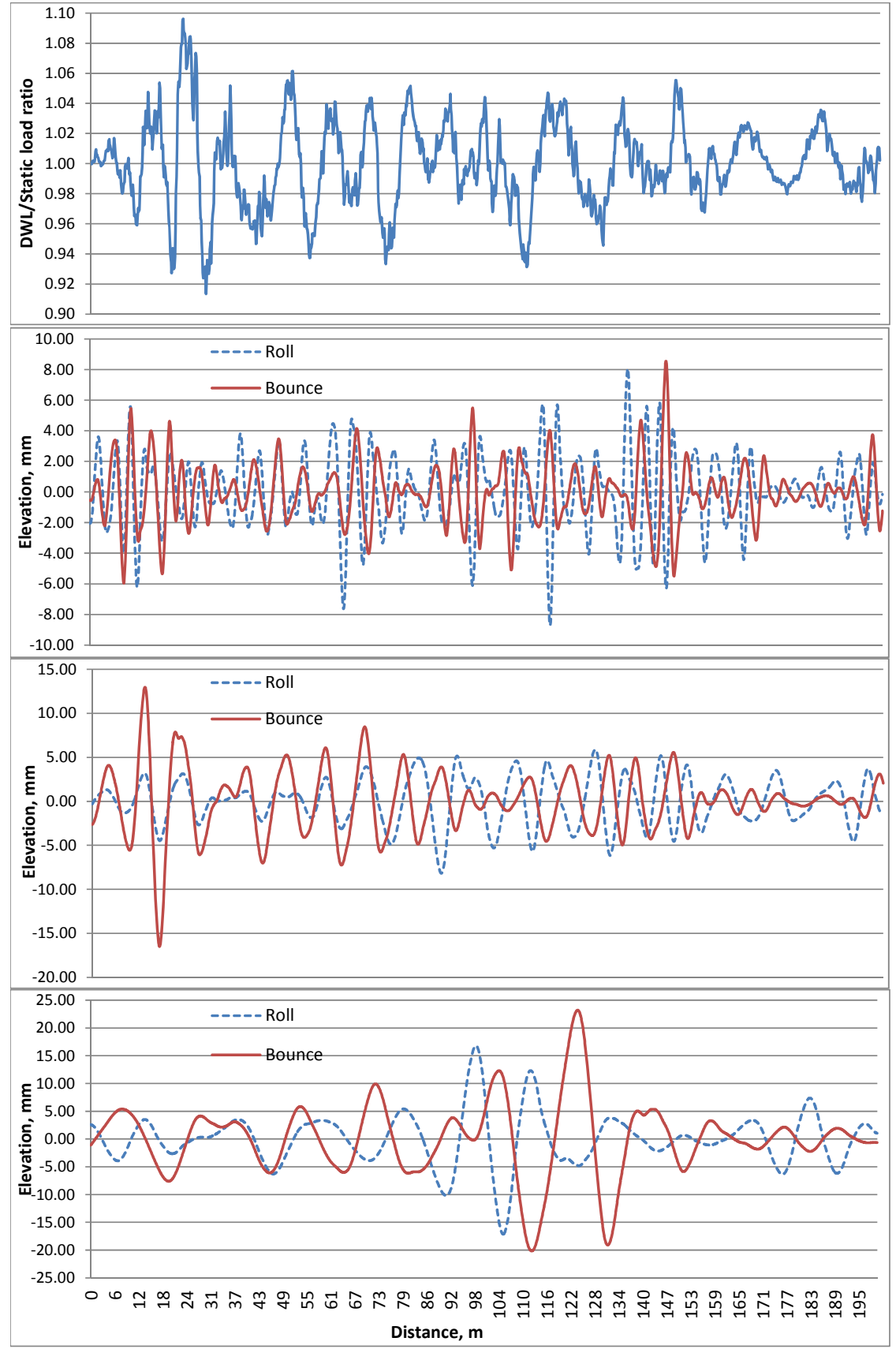

Figure 3: DWL to static load ratio and roll and bounce excitation inputs in D6, D7 and D8 respectively for section No. 10. 
- The variations in elevations and DWL signals are lower for sections with lower HATI values. For example, the range of DWL to static load ratio for section 2 (HATI $=0.58$ ) is $0.96-1.03,0.95-1.07$ for section 7 (HATI = 1.44) and 0.91-1.1 for section 10 (HATI = 3.09).

- Both roll and bounce excitation inputs are evident in all subbands for the three sections. Figure 4 presents the filtered elevation profiles of both wheel paths for section 7. Comparing figure 4 with figure 2, it can observed that the roll excitation input in all three subbands coincides with changes in movement of direction between the two wheel paths (highlighted with circles). This is when the outer profile (OTElev) changes from being higher than the inner profile (INElev) to being lower or vice versa. Also, the roll excitation input is higher when the differences in elevations between the wheel paths are high. The same trends were observed for sections 2 and 10 .

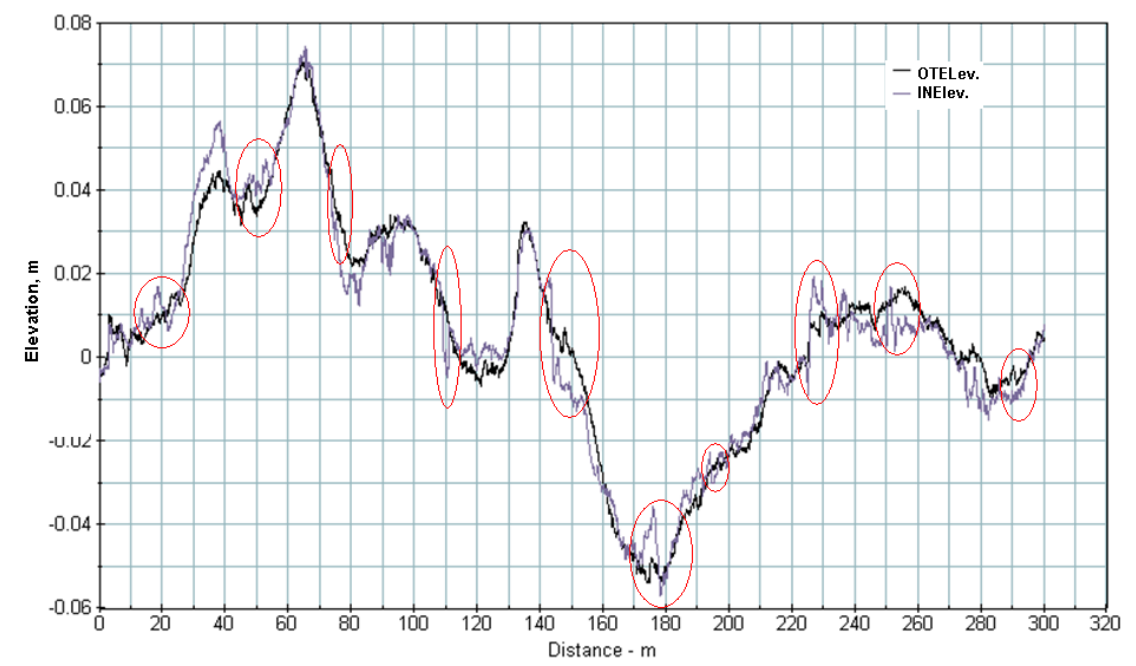

Figure 4: Elevation profile in the outer and inner wheel paths for test section No. 7.

The findings presented above confirm that there are contribution to DWL from excitations of body bounce and roll which confirm the observations from DWL spectra. The limited testing also indicates that using wavelet analysis is useful in identifying and locating the sources of DWL excitations. This type of assessment is suitable at project level for sections detected by HATI i.e. have HATI values greater than the threshold.

\section{Discussion and conclusions}

Pavement damage due to DWL is the result of the dynamic interaction of a mix of heavy vehicles fleet and surface roughness. Hence, it could be argued that the 
vehicle used in simulating DWL in this study is only one type in this mix and that the findings of the study may not be applicable to a typical fleet mix. The authors would suggest that HATI would also be a good representative of the damaging potential of a mix of vehicles. At normal highway speeds, roughness wavelengths that cause excitation of low and high frequency modes of all suspension types of heavy vehicles fall in the range to which HATI filter is sensitive. HATI filter captures the contributions of both short and long roughness wavelengths but with higher sensitivity to the latter, which has proven to have higher contribution to DWL. Further, HATI was developed using a mix of prime-mover semi-trailer combination units with different ages, suspension systems and other properties. However, further testing is required to confirm the suitability of HATI at different speeds and operating environments. It should include a larger sample size of sections covering a wide range of operating and environmental conditions. Additionally, simulation models should be representatives of the current trends in the heavy transport industry i.e. longer and heavier trucks such as B-Doubles.

The findings from the analyses presented in this paper have shown that, for the sections tested herein, the highest contribution to the DWL generated at the truck tyre-pavement interface is from the low frequency vibration modes namely; body bounce, pitch and roll. These findings are confirmed with wavelet analysis which has proven to be useful in identifying and locating the sources of DWL excitation inputs along the pavement sections tested herein. The approach proposed herein, when confirmed with further testing, would be useful to road agencies for managing their pavement assets at both network and project levels. It would help them identify problem sections, locate and identify the sources of excitation and take appropriate measures to control or eliminate the accelerated wear due to DWL.

\section{References}

[1] Sweatman, P.F., A Study of the Dynamic Wheel Forces in Axle Group Suspensions of Heavy Vehicles, ARRB Special Report No. 27, Melbourne, Australia, 1983.

[2] Cebon, D., Handbook of Vehicle Road Interaction, Swets and Zeitlinger publishers, London, 1999.

[3] OECD, Dynamic Interaction of Vehicle and Infrastructure Experiment (OECD IR6 Project: Dynamic Interaction of Heavy Vehicles with Roads and Bridges, DIVINE Project), Proceedings of the Asia-Pacific Concluding Conference, Melbourne, Australia, 1997.

[4] De Pont, J., Road Profile Characterisation, Transit New Zealand Research Report No. 29, 1994.

[5] Papagiannakis, A.T. and Gujarathi, M.S., Roughness Model Describing Heavy Vehicle-Pavement Interaction, Transportation Research Record 1501, pp. 50-59, 1995. 
[6] Sweatman, P. and Addis, R., Improving the Interaction between Heavy Trucks, Roads and Bridges, 5th International Symposium on Heavy Vehicle Weights and Dimensions, Australia, 1998.

[7] Hassan R and McManus K, Sustainable Mobility for Heavy Freight Vehicles, International Journal of Sustainable Development and Planning, Vol. 5, No. 3, WIT Press, UK, 2010.

[8] Cole, D.J. and Cebon, D., Influence of Tractor-Trailer Interaction on Assessment of Road Damaging Performance, Proceedings of the Institution of Mechanical Engineers, Part D: Journal of Automobile Engineering, 212:1, SAGE publishers, 1998.

[9] Bernard, J. and Dolcemascolo, V., Dynamic Interaction between Instrumented Vehicles and Pavements, 5th International Symposium on Heavy Vehicle Weights and Dimensions, Australia, 1998.

[10] Doré, G., Flamand, M. and Pascale, P. Analysis of the wavelength content of the longitudinal profiles for C-LTPP test sections, Canadian Journal of Civil Engineering, Volume 29, Issue 1, Canada, pp. 50-57, 2002.

[11] Brown, D. Liu, W. and Henning, T.F.P., Identifying pavement deterioration by enhancing the definition of road roughness, Research Report 430. NZTA, NZ Transport Agency Wellington, 2010.

[12] Delanne, Y. and Pereira, P.A. A., Advantages and limits of different road roughness profile signal-processing procedures applied in Europe, Transportation Research Record, No1764, USA, pp: 254-259, 2001.

[13] Liu, W. and Fwa, T.F., Application of wavelet transform analysis for pavement roughness studies, Proceedings of the Eighth International Conference on Applications of Advanced Technologies in Transportation Engineering, Beijing, pp: 455-459, 2004.

[14] Papagiannakis, A.T., Zelelew, H.M., and Muhunthan, B., A wavelet interpretation of vehicle-pavement interaction. International Journal of Pavement Engineering. Volume 8, Issue 3, pp. 245-252, 2007. 